\title{
Real-time measurement of luminal compliance during transoral incisionless fundoplication
}

A 71-year-old woman who had a family history of esophageal cancer presented with symptoms of chronic heartburn. The patient expressed interest in definitive treatment, declining long-term medical therapy and opting to undergo transoral incisionless fundoplication (TIF) ( $\bullet$ Fig. 1 ). This was accomplished by using the EsophyX Device (EndoGastric Solutions, San Mateo, California, USA). Before and after the TIF procedure, an EndoFLIP Endolumenal Functional Lumen Imaging Probe (Crospon, Carlsbad, California, USA) was used to measure the luminal compliance and high pressure zone of the gastroesophageal junction. EndoFLIP utilizes impedance planimetry technology to measure the geometry and physical properties of hollow lumina. The patient was observed for 24 hours after the procedure and was discharged in a stable condition. Recent prospective randomized studies have shown the TIF procedure to be effective treatment for a subset of patients with acid reflux [1,2]. However, although TIF appears to be a very promising therapeutic option for patients who refuse to take medications or whose condition is refractory to medical therapy, some patients may not respond. Up to one-third may have persistent symptoms after 6 months, and up to $11 \%$ have no response at all [2]. Real-time feedback of the morphologic and structural effects of TIF at the gastroesophageal junction and high pressure zone may be helpful in predicting the efficacy of a TIF procedure.

The results of EndoFLIP measurement before and after the TIF procedure in this patient confirmed a decrease in luminal distensibility (from 35 to $31 \mathrm{~mm}^{3} / \mathrm{mmHg}$ ) and an increase in the length of the high pressure zone (from approximately 1.5 to $2.5 \mathrm{~cm}$ ) ( Fig. 2). Clinically, the patient responded well after the procedure. EndoFLIP measurement during TIF may guide endoscopists as to the adequacy of a fundoplication, predict the clinical response, and thereby improve patient outcomes and wrap durability. Prospective studies are needed to confirm this notion.
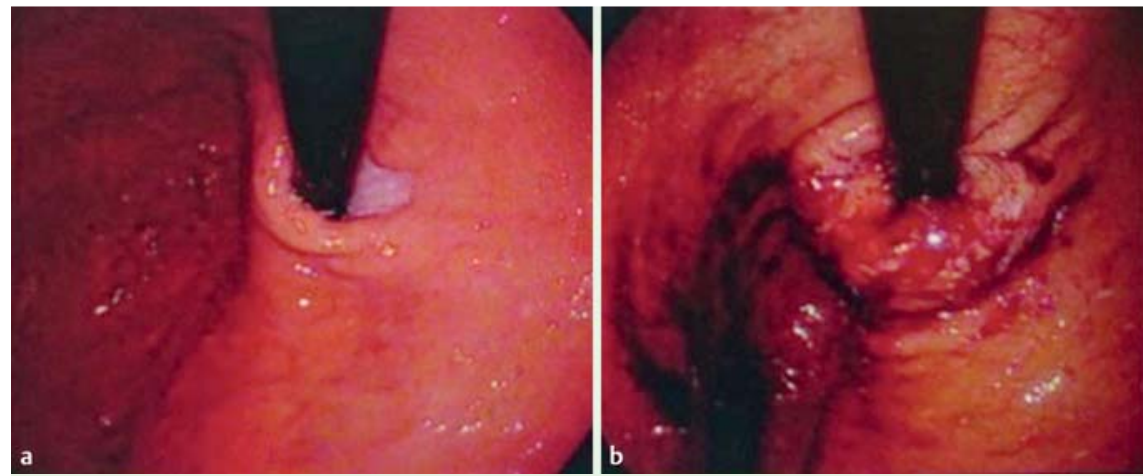

Fig. 1 Transoral incisionless fundoplication in a 71-year-old patient with symptoms of chronic heartburn and a family history of esophageal cancer. Retroflexed endoscopic views of the gastric cardia: a before fundoplication; $\mathbf{b}$ after fundoplication.

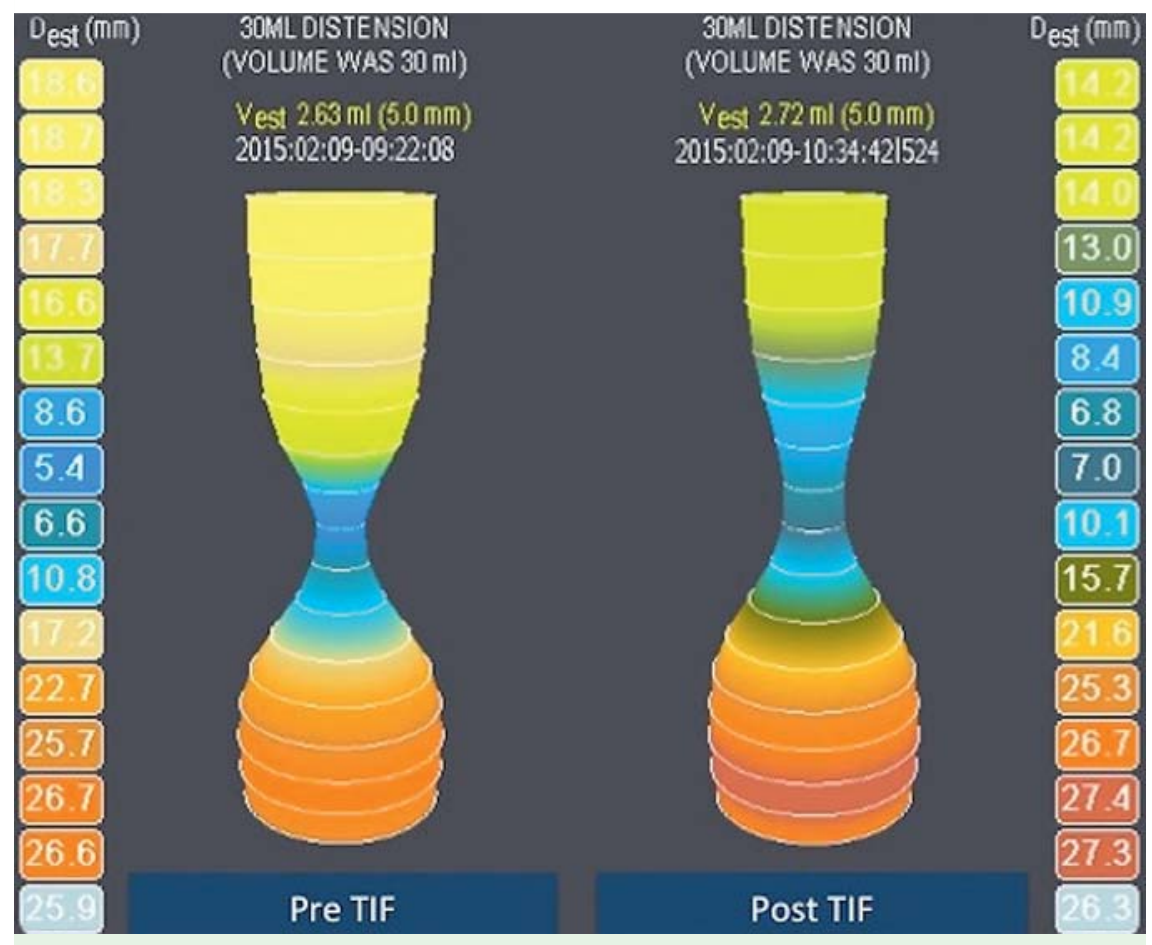

Fig.2 Results of EndoFLIP measurement at the level of the gastroesophageal junction before and after transoral incisionless fundoplication (TIF).

Endoscopy_UCTN_Code_TTT_1AO_2AJ

Competing interests: None
Sheba Vohra ${ }^{1}$, Vincent Kuo ${ }^{1}$, Bahar Madani ${ }^{1}$, Reem Sharaiha², Michel Kahaleh², Paul Tarnasky", Prashant Kedia ${ }^{1}$

${ }^{1}$ Department of Gastroenterology, Methodist Dallas Medical Center, Dallas, Texas, USA

2 Division of Gastroenterology and Hepatology, Weill Cornell Medical College, New York, New York, USA 


\section{References}

1 Trad KS, Barnes WE, Simoni G et al. Surgical innovation effective in eliminating GERD symptoms in partial responders to proton pump inhibitor therapy at 6 months: the TEMPO randomized clinical trial. Surg Innov 2015; $22: 26-40$

2 HunterJG, Kahrilas PJ, Bell RCW et al. Efficacy of transoral fundoplication vs omeprazole for treatment of regurgitation in a randomized controlled trial. Gastroenterology 2015; 148: 324-333.e5
Bibliography

Dol http://dx.doi.org/

10.1055/s-0034-1391907

Endoscopy 2015; 47: E282-E283

(c) Georg Thieme Verlag KG

Stuttgart · New York

ISSN 0013-726X
Corresponding author

Prashant Kedia, MD

Department of Gastroenterology Methodist Dallas Medical Center Dallas, Texas 75208

USA

Fax: +1-214-943-5871

kediaps@gmail.com 